(Aus der neurologischen Abteilung des Allerheiligen-Hospitals zu Breslau [Primärarzt: Prof. Dr. O. Foerster].)

\title{
Untersuchungen am Nervus opticus mit Leducschem Strome.
}

\author{
Von \\ Hanns Taterka, \\ Volontärarzt der Abteilung.
}

(Eingegangen am 9. Februar 1921.)

Die Zahl der Impulse, die der motorische Nerv dem Muskel erteilt, beträgt etwa 50 bis 100 in einer Sekunde. Da nun bei den hochfrequent unterbrochenen galvanischen Gleichströmen niederer Spannung nach Led $\mathrm{uc}^{1}$ ) die günstigste Unterbrechungsziffer zwischen 80 und 100 Unterbrechungen in der Sekunde liegt, so geht schon aus diesem Umstand hervor, da $\beta$ dieser Strom dem physiologischen Muskelaktionsstrom am nächsten kommt und dadurch einen wesentlichen Vorzug vor den anderen therapeutisch angewandten Strömen besitzt. Der Leduesche Unterbrecher ermöglicht es ferner, mit Leichtigkeit Stärke und Spannung des Stromes und somit auch zu jeder Zeit den Widerstand des Patienten, sowie die Zahl der Schließungen und Unterbrechungen in der Sekunde genau anzugeben. Weiterhin kann man bei diesem Unterbrecher leicht und ge na u die Zeitdauer der Stromschließung während jeder Stromperiode regulieren und messen.

Das Wesen der Galvanisation nach Led ue liegt in der Einschaltung eines rhythmischen Unterbrechers mit hoher Unterbrechungszahl in den Stromkreis. Das geschieht am besten durch eine mit der Motorachse verbundene rotierende Scheibe, die vier durch Isoliermateial getrennte Silbersektoren trägt. Auf dieser schleifen zwei Stromabnehmer, von denen der eine gegen den anderen verstellbar ist. Die Zeit, die ein Sektor braucht, um in seiner ganzen Ausdehnung an einem Abnehmer vorbeizupassieren, bezeichnet man als eine Stromperiode. Der bewegliche Abnehmer kann so gestellt werden, da $B$ beide Abnehmer während der ganzen Periode gleichzeitig auf demselben Segmente schleifen, $\mathrm{da} \beta$ also der Strom während der ganzen Periode geschlossen bleibt. Durch Entfernung des beweglichen Abnehmers von dem festen kann man den Unterbrecher so einstellen, daß der Strom nur während be- 
stimmter, auf einer Skala ablesbarer Teile der Periode geschlossen bleibt und während der reziproken Teile der Periode unterbrochen ist.

Die eigentümliche Wirkung des Stromes erzeugt eine Hypästhesie der durchströmten Partie. Periphere Nervengebiete im Stromkreise werden unterempfindlich. Das Zentralnervensystem reagiert analog mit einem teilweisen Funktionsausfall, der sogenannten „Elektronarkose ${ }^{\text {(2) }}$ ). Der Leducsche Strom erregt ähnlich wie der galvanische den Muskel direkt und indirekt. Degenerierte Muskeln können sogar mitunter noch zucken, wenn auf gewöhnliche galvanische Reizung keine Kontraktion mehr auftritt. An den Stellen, an welchen sich die Elektroden befinden, kommt es zu Hyperämie, Rötung und Erwärmung, die die Applikationszeit lange überdauern. Bei empfindlichen Personen wurde bereits nach drei Minuten bei einer Stromstärke von 3 M. A. lokale Exsudation (Quaddelbildung) beobachtet. Die Wirkung der Kathode ist stärker als die der Anode.

Es besteht also eine Trias der Stromwirkung [D uschak k)]: Hypästhesierung, Hyperämisierung und gleichzeitige energische Muskelreizung.

Bisher wurde der Strom angewandt bei Migräne, Neuralgien, chronischen Neuritiden, sowie zur Behandlung von Hyperästhesien und Lähmungen bei peripheren Nervenverletzungen. Auch Schlafstörungen wurden günstig beeinflußt.

Es ist also bisher die Wirkung des Leducschen Stromes auf das Zentralnervensystem und auf das periphere Nervengebiet festgestellt und der Therapie nutzbar gemacht worden. Noch nicht untersucht ist dagegen seine Wirkung auf die sensorischen Nerven.

Bevor ich nun zu meinen Untersuchungen übergehe, will ich zweckmäßig erst einige Worte über die elektrische Erregung von Sinnesorganen vorausschicken.

Analog dem Zuckungsgesetz für motorische Nerven und Muskeln antwortet auch der sensorische Apparat in gesetzmäßiger Weise auf die einzelnen Reizmomente. Die Reaktion besteht beim Auge in einem (gewöhnlich farbigen) Lichtbild, beim Ohr in einem Klang, bei Erregung der Geschmacksknospen in einem sauren Geschmack; hierzu kommt noch die bekannte elektrische Erregbarkeit des statischen Organs im Mittelohr.

Die wohl zeitlich mit am weitesten zurückliegenden Beobachtungen Erdmanns $s^{4}$ ) über elektrische Opticusreizung ergaben beim Stromschluß eine blitzartige, blendende lichterscheinung. Der dem Auge angelegte positive Pol erregte eine bläulichviolette Lichterscheinung, der negative Pol dagegen ein rötlichgelbes Licht, das in kreisförmiger Gestalt das ganze Gesichtsfeld erleuchtete. Gelegentlich war es schwer, das durch die Lichterscheinung erregte Nachbild von dieser selbst 
zu trennen. - Auch hinsichtlich der Erregang anderer Sinnesnerven werden von demselben Autor schon einige Beobachtungen mitgeteilt. So wird der elektrische Geschmack an der positiven Elektrode als deutlich sauer, an der negativen als laugenhaft, herb, alkalisch beschrieben. Das Ohr reagierte mit verschiedenartigen Geräuschen, deren Klangcharakter nur ungenau festgestellt wird.

Diese und auch einige der folgenden Beobachtungen lassen noch fast völlig jede Gesetzmäßigkєit in der Versuchsanordnung vermissen, ebenso die Möglichkeit, die gewonnenen Ergebnisse mit den feststehenden Erregungsgesetzen am motorischen Nerven zu vergleichen und evtl. in Ubereinstimmung zu bringen. Daraus und vielleicht auch aus der Verschiedenheit der Versuchsanordnung lassen sich wohl die zum Teil erheblich differenten Resultate erklären.

Schliephake ${ }^{5}$ ) fand bei seinen Untersuchungen an sich selbst folgende Wirkung des galvanischen Stromes:

1. Bei Schließung des aufsteigenden und bei Öffnung des absteigenden Stromes erscheint im ersten Moment das ganze Sehfeld bläulichviolett erleuchtet, nach wenigen Sekunden verkleinert sich das $\mathrm{Phä-}$ nomen zu einer zentralen hellen Scheibe. Bei Öffnung des auf- und bei Schließung des absteigenden Stromes erscheint im bläulich-violetten Sehfelde eine dunkle Scheibe.

2. Objektive Farben werden bei Schließung des aufsteigenden und bei Öffnung des absteigenden Stromes $\epsilon$ twa so verändert, als ob Violett hinzugefügt wäre, bei Öffnung dєs auf- und Schließung des absteigenden Stromes in dem Sinne, als ob $\mathrm{G} \mathrm{lb}$ hinzugefügt wäre.

3. Die Wahrnehmung äußcrer Objekte wird durch die Einwirkung des galvanischen Stromes beeinflußt, und zwar in der Weise, daß, wenn der Strom Erhellung des Grundes, auf welchem dunkle Objekte sich befinden, bewirkt, diese Objekte deutlicher, wenn er Verdunkelung des Grundes bewirkt, die Objekte undeutlicher erscheinen.

G. E. Müller ${ }^{6}$ ) fand, daß die durch den aufsteigenden oder absteigenden galvanischen Strom ausgelösten Empfindungen die Empfindungen von Gegenfarben seien; und zwar bewirke bei geöffnetem Auge der aufsteigende Strom eine Verstärkung der Weißerregung und eine Schwächung der Schwarzerregung, während der absteigende Strom die umgekehrte Wirkung habe. Bei aufsteigendem Strome sei die Farbe der galvanischen Gesichtsempfindung ein nach Rot hinneigendes Blau, bei entgegengesetzter Stromrichtung grüngelb. Die den beiden Stromrichtungen entsprechenden Empfindungen sind also Empfindungen von Komplementärfarben. Der aufsteigende Strom wirkt stärker und ausgeprägter als der absteigende. Die Netzhautschicht, auf welche der Strom einwirke, sei wahrscheinlich die der Stäbchen und Zapfen, die Sehnervenfasern würden jedenfalls nicht direkt gereizt. 
Nach Finkelstein ${ }^{7}$ ) sind wir imstande, durch starke elektrische Reizung der Netzhaut die Abbildung unseres eigenen Gesichtsfeldes hervorzurufen. Das ganze Gesichtsfeld sei als mosaikartige Fläche zu sehen.

Nach v. Helmholz ${ }^{8}$ ) läßt die elektrische Reizung sich auch auf einzelne Teile der Netzhaut beschränken. Elektrische konstante Durchströmung der Retina in der Richtung von den Zapfen zu den zugehörigen Ganglienzellen gibt die Empfindung von Dunkel, die entgegengesetzte Durchströmung gibt die Empfindung von Hell.

Neftel7) fand die galvanische Reaktion des Sehnerven bei verschiedenen Individuen sehr verschieden. Bei einem Patienten erfolgte auf Applikation der schwächsten Ströme an irgendeinem Teile des Kopfes die Wahrnehmung von schönem Blau unter dem Einfluß der Kathode, von Violett unter Einwirkung der Anode. Auf stärkste Ströme erfolgte kein Lichtblitz. Bei anderen genügten schwächste Ströme, um starke Lichtblitze hervorzurufen, während stärkste Ströme keine Farbenwahrnehmung erzeugten.

Tarducci ${ }^{9}$ ) fand bei aufsteigendem Strome (Kathode im Nacken), der zehn Minuten mit 3 M. A. floß, daß

1. das Gesichtsfeld für Weiß und Farben erheblich vergrößert war,

2. daß diese Erweiterung schnell eintritt, ihr Maximum aber erst am 3. Tage erreicht und nach 9 Tagen verschwunden ist,

3. daß dieser Einfluß am meisten bei Grün, demnächst bei Blau und am wenigsten bei Weiß hervortritt;

4. daß die Ausdehnung nach unten und außen am bedeutendsten ist,

5. daß gleichzeitig dieselbe Wirkung am anderen Auge auftritt.

Absteigender Strom ergab unter gleichen Bedingungen fast den umgekehrten Erfolg.

Diese Angaben Tarduccis sind von anderen Autoren im wesentlichen nicht bestätigt worden und bedürften hinsichtlich der Dauer der Wirkung wohl besonders einer Nachprüfung. Utberhaupt scheinen die oben beschriebenen Lichtphänomene mehr auf einer Reizung der Netzhaut zu beruhen als auf einer direkten Erregung des Sehnerven. Auch Sile $\mathbf{x}^{10}$ ) glaubt, daß die bekannten Lichterscheinungen beim Öffnen und Schließen des Stromes von der Netzhaut ausgehen, daß aber auch der Sehnerv selbst $u$. U. den Lichtblitz auslösen kann. Die Erweiterung des Gesichtsfeldes für farbige Objekte kann er auch nicht bestätigen.

Erst Brenners ${ }^{11}$ ) Untersuchungen am N. acusticus verdanken wir größere Klarheit auf dem Gebiete der elektrischen Erregung von Sinnesnerven. Brenner hat zuerst ein bestimmtes Gesetz gefunden, das das Verhältnis von Elektrizität zum Hörnerven betrifft, und das Aufschluß gab, in welcher Weise e in lebender Sinnesnerv durch Elek- 
trizität erregungsfähig sei. Dieses Gesetz bezeichnet man als die sogenannte „Reaktionsformel des N. acusticus“. Setzt man die Kathode einer elektrischen konstanten Kette nahe dem äußeren Gehörgang - auf den Tragus - auf, - die Anode kann auf irgendeinen anderen beliebigen Körperpunkt aufgesetzt werden - so erfolgt beim Schließen der Stromkette eine Klangwahrnehmung. Diese bleibt zwar bei weiterer elektrisch-konstanter Einwirkung noch eine Zeitlang bestehen, um aber dann allmählich abzusinken und ganz aufzuhören, wenn durch Kathodenöffnung die Stromkette geöffnet wurde. Den drei Phasen der Stromapplikation entsprechen also auch drei durch den N. acusticus vermittelte Phänomene:

1. Bei Kathodenschlu $B$ - Klangentstehung.

2. Bei Kathodenstromdauer - Klangdauer und Abklingen.

3. Bei Kathodenöffnung - Klangfehlen.

Bei umgekehrter Stromanordnung (Anode auf dem Tragus, Kathode beliebig) ergab sich:

1. Bei Anodenschluß - kein Klang.

2. Bei Anodendauer - ebenfalls kein Klang.

3. Bei Anodenöffnung - schwache Klangempfindung.

Brenner fand weiterhin, daß man je nach der Zahl der Elemente eine primäre, sekundäre und tertiäre Erregbarkeit des N. acusticus zu unterscheiden habe. Primär von 3 Elementen an aufwärts. Wenn man bald darauf den Strom abschwächt, so zeigt sich sekundäre Erregbarkeit. Weitere Schwächung ruft keinen Klang mehr hervor, aber Stromwendung zeitigt zuweilen noch eine Klangwirkung. (Tertiäre Erregbarkeit.) Stromwendung ist somit der wirksamste Reiz für den N. acusticus.

Die Brennersche Normalformel gestattet nicht nur, diagnostisch Anomalien des N. acusticus festzustellen, sondern gibt auch therapeutische Hinweise bei nervösem Ohrensausen und Torpor des Gehörsnerven, je nachdem der Strom vermindernd oder vermehrend auf das Ohrensausen wirkte oder gar nicht.

Auch $\mathrm{Erb}^{12}$ ), der wichtige Beiträge zu diesem Gebiete geliefert hat, hält die Brennerschen Versuche für bedeutungsvoll und therapentisch außerordentlich ergiebig.

Später sind dann auch für den Augennerven in bezug auf sein Verhalten zum elektrischen Strome ganz genaue Gesetze aufgestellt worden, und zwar von Richter ${ }^{13}$ ), der zu diesem Zwecke ein neues Elektrodenpaar für das Auge (nebst solchen für andere Sinnesorgane) angegeben hat. Die eine Elektrode ist stabförmig und endet vorn in einem tellerförmigen Aufsatz, der der Bulbusform angepaßt ist. Die andere stellt einen dünnen, biegsamen Leitungsdraht mit knopfförmigem Ende dar und wird durch die Nase - nach Art des Ohr- 
katheters - vorgeschoben und dann gedreht, bis der Knopf das Rachendach berührt. Das Neue und Wichtige dieser Anordnung besteht nun darin, daß der Bulbus direkt in den Stromkreis eingeschaltet ist. Bei diesen Versuchen Richters zeigte sich, wenn sich die Kathode auf dem Bulbus, die Anode am Rachendach befand, folgendes Gesetz für die elektrische Reizung des N. opticus durch den konstanten Strom: „Der N. opticus wird durch schwache und starke absteigende Ströme n ur derart gereizt, daß weder bei Kathodenschluß noch bei Kathodendauer eine Lichterscheinung eintritt, da $B$ dagegen $n$ ur bei Kathodenöffnung ein helles, homogen erleuchtetes, an Farbe dem elektrischen Licht gleichendes Gesichtsfeld entsteht." Bei umgekehrter Stromanordnung (Kathode am Rachendach, Anode auf dem Bulbus) geben schwache Ströme von 2 Volt keine Reaktion, Ströme von 4 Volt undeutliche, und erst Ströme von 6 Volt zeigen deutliche, von der Kathodenreaktion total verschiedene Reaktion: „Anodenschließung des aufsteigenden Stromes löst eine stern- und punktförmige zentrale, bläulichgrüne Lichterscheinung aus, welche von einem peripheren, matteren Lichtkreis umgeben ist; das intermediäre Feld ist schwächer erleuchtet. Diese Lichterscheinung schwächt sich bei Anodendaner nur etwas ab und macht bei Anodenöffnung einem tiefschwarzen Gesichtsfeld Platz, das nur am Anfang des Verlöschens von radiären, kurzen, peripheren, hellen Lichtfransen umringt ist." Dieses Richtersche Opticusgesetz sagt nun gerade das Gegenteil von der Brennerschen Reaktionsformel des $\mathrm{N}$. acusticus, und zwar insofern, als die Kathodenwirkung bei ersterem der Anodenwirkung bei letzterem entspricht, und umgekehrt.

Diesen Gegensatz der Ergebnisse - andere Beobachter sollen übrigens auch völlige Umkehr der Br e $n$ n e r schen Formel gefunden haben - führt Richter möglicherweise auf die Verschiedenheit der Versuchsanordnung zurück. Seine Anordnung schaltet den N. opticus in seiner Längsrichtung in den elektrischen Strom, was bei Brenner am N. acusticus nicht der Fall war; außerdem war bei Brenner die nächste Annäherung an den Acusticusendapparat noch etwa $4 \frac{1}{2} \mathrm{~cm}$ - die Länge des äußeren Gehörganges und der Paukenhöhle - von letzterem entfernt.

Späterhin hat dann Richter ${ }^{11}$ ) seine Versuche fortgesetzt und versucht, neue Gesetze vergleichender Natur über die Erregung der Sinnesnerven und motorischen Nerven zu gewinnen.

Zunächst erörtert er wieder das in seinen Vorarbeiten bereits festgelegte Gesetz für die Erregung des N. opticus mittels des konstanten Stromes, das im wesentlichen nur eine Modifikation des bereits früher von ihm angegebenen darstellt. Er legt in dieser Arbeit besonderen Wert auf genaue quantitative sowie vergleichende Angaben und berücksichtigt 
die Qualität der Lichterscheinung erst in zweiter Linie. Neu und bedeutungsvoll sind seine Untersuchungen über die Nacherregung. Das Opticusgesetz lautet: Bei einsteigenden Strömen (Anode auf dem Bulbus, Kathode hinter dem Auge am Rachendach) antwortet der N. opticus so lange mit Lichtreizung, bis der Reiz wieder aufhört. Es glimmt dann sichtbar im Auge die Nervenpapille in bläulich-weißem Licht, bis der Strom wegfällt; alsdann tritt tiefe Dunkelheit ein. - Bei Wirkungseintritt des aussteigenden Stromes (Kathode auf dem Bulbus, Anode hinter dem Auge) zeigt die Retina das Bild tiefster Dunkelheit, bis beim Öffnen des aussteigenden Stromes unter einem nochmaligen Lichtblitz der Stromreiz optisch aufhört. Während aber bei der ersten Versuchsanordnung keine Nacherregung sichtbar wurde, zeigt sich bei der zweiten Versuchsanordnung ein ganz bestimmtes Nacherregungsgesetz, nämlich, es tritt als Nacherregung die Papille leuchtend in Erscheinung. Da nun nicht anzunehmen ist, daß bei einsteigenden Strömen eine Nacherregung fehlt, so sei eben die absolute, nachher eintretende Dunkelheit das Gesetz der Nacherregung und Erholung. Dagegen sei bei aussteigenden Strömen das Sichtbarwerden der Sehnervenpapille als Nacherregungsgesetz bzw. Ausgleichsgesetz hinzustellen. Dieselben Gesetze werden noch deutlicher, wenn man die Anode auf den Bulbus setzt und die Kathode - in Form eines konisch abgestumpften Metallstabes - anal verlegt. Bei beiden Stromrichtungen läßt sich außerdem feststellen, daß die Reizwirkung vom An- oder Kathelektrotonus unabhängig ist, sondern daß diecer Sinnesnerv von der Reizleitung abhängt, $d . h$. also von einsteigenden oder aussteigenden Strömen beeinflußt wird, daß er ferner auf einsteigende Ströme während der ganzen Reizdauer sinngemäß mit einer normalen Lichterscheinung antwortet, $d a ß$ er hingegen auf aussteigende Ströme nicht antwortet, außer bei ihrem Eintritt und Austritt. Diese letzteren Ströme bezeichnet Richter als perverse oder antiphysiologische. -

In gleicher Weise wie oben wurde auch am $\mathrm{N}$. glossopharyngeus und am $\mathrm{N}$. olfactorius experimentiert; letzterer ist am schwersten zu beurteilen und zu treffen. Auch diese Ergebnisse entsprechen in analoger Weise dem für den $\mathrm{N}$. opticus gefundenen Gesetze.

Anschauungen anderer Autoren - die Literatur über elektrische Erregung der Nn. I. und IX. ist sehr gering - weichen zum Teil recht erheblich von den Richter schen ab. Am nächsten kommen ihm Hoffmann und Banzel15), deren Versuche am N. IX., in die Richtersche Versuchsanordnung übertragen, annähernd ein analoges Resultat ergeben dürften. Nach diesen Autoren beruht der elektrische Geschmack auf einer Kombination von Produkten der Elektrolyse und elektrischer Reizung des Sinnesnerven. v. Zeynek $\left.{ }^{16}\right)$ findet, daß sich die Geschmacksempfindung mit der Spannung ändert und folgert daraus, 
daß der elektrische Geschmack lediglich von der Elektrolyse des Speichels herrühre, zumal bei festem Aufdrücken der Elektrode auf die Zunge unter 2 Volt kein deutlicher Geschmack zu empfinden war. He uman $n^{17}$ ) erklärt die Wirkung des elektrischen Stromes im Tierkörper nur mit einer Verschiebung der Ionen des Körpers und einer damit im Zusammenhang stehenden eventuellen Konzentrationsveränderung an den Zellmembranen. Die Muskelkontraktionen bei elektrischer Reizung eines motorischen Nerven, die Gefühlswahrnehmungen, welche bei Durchgang des Stromes in der Haut vorkommen, die Lichtwahrnehmung bei Durchgang des Stromes durch das Auge, der Voltaschwindel bei Durchgang des Stromes durch die halbzirkelförmigen Kanäle und schließlich der elektrische Geschmack; alles sei nichts anderes als Einwirkung der Ionenbewegung, d. h. Einwirkung einer in hohem Grade verstärkten Diffusion nach den Organzellen zu, von dem einen oder anderen Bestandteile der dem Organ am nächsten befindlichen Salzlösung. Nach Heumann werden nicht die Nerven, sondern die empfindungsauslösenden Endorgane gereizt. Bei dieser Theorie berücksichtigt der Autor jedoch überhaupt nicht das Pfl üg er sche und Du-Bois-Raymondsche Gesetz, deren Gültigkeit wenigstens für den motorischen Nerven - wohl unbestritten sein dürfte.

Der N. acusticus ist nach Richter einer galvanischen Reizung überhaupt nicht unterstellbar, höchstens sein $\mathrm{N}$. vestibularis-Teil. Nicht ein einziges Mal antwortete der $\mathbf{N}$. acusticus, auch nicht einmal bei feuchter Zuleitung bis aufs Trommelfell mit einem Klang, sondern nur der Vestibularapparat war imstande, auf den elektrischen Reiz zu reagieren. Richter erklärt dieses Verhalten damit, daß der galvanische Strom kein adäquater Reiz zur Auslösung einer Tonempfindung sei. Dazu gehörten eben ganz spezifische Klangwellen und nicht elektrische Wellen, die zwar imstande seien, den Opticus zu erregen wegen ihrer Verwandschaft zu Lichtwellen, aber nicht den N. acusticus.

Zusammenfassend fand also Richter, daß der Sinnesnerv durch einsteigende Ströme während des ganzen Zeitverlaufes gereizt wird, daß er dagegen durch aussteigende Ströme nur im Anfang Reizung zeigt, dann eine Unterbrechung aufweist durch absolute Nichtbeantwortung des Reizes und beim Aufhören des Reizes in eine positive Nacherregung übergeht. Untersuchungen am motorischen Nerven ergaben jedoch gerade das umgekehrte Resultat.

Es stehen sich somit zwei große Kategorien von Nervengesetzen diametral gegenüber, und zwar findet Richter,

1. daß das Zentralorgan des Gehirns oder Rückenmarks nur als Reizleiter an den Gesetzen teilnimmt, daß aber die Austragung des 
Reizes selbst den teleologischen Endorganen der Sinnesnerven oder motorischen Nerven vorbehalten ist;

2. der Sinnesnerv kann durch Stromrichtungen, welche den physiologischen Reizrichtungen entsprechen, zu Dauererregungen angeregt werden, also hier einsteigenden Strömen;

3. gerade umgekehrt verhalten sich die motorischen Nerven, welche nur durch aussteigende Ströme ,entsprechend der Willensrichtung“ bzw. der Richtung des motorischen Reizes zu Dauerwirkung und Dauerarbeitsleistung angeregt werden können.

Was also des Sinnesnerven physiologisches Dauererregungsgesetz ist, bedeutet für den motorischen Nerven das gegensinnige Gesetz, und umgekehrt.

Der sensible Nerv schließt sich den Gesetzen der Sinnesnerven ungefähr an, nur daß seine Erregungskurve keine Dauererregung zuzulassen scheint, sondern einen schräglinigen Abfall seiner Erregungshöhe herbeizuführen sucht. -

Die oben beschriebenen Erscheinungen der Nacherregung lassen sich auch gesetzmäßig zusammenfassen. Richter formuliert sie folgendermaßen:

1. Der in Reizzustand versetzte Nerv gerät nach Aufhören des Reizes in Nacherregung, und zwar ist die Nacherregung für im physiologischen Sinne sich abwickelnde Reize das Gesetz der Erholung.

2. Auf Reize, welche sich im antiphysiologischen Sinne abwickeln, gerät der Nerv in eine Nacherregung, welche erst wieder das ursprüngliche normale Reizgesetz herbeiführt und erst nach dessen Abklingen den Erholungsgesetzen unterliegt.

3. Die Nacherregung ist durchaus proportional der Reizstärke; dagegen ist sie im normalen, nicht pathologischen Nerven für schwache oder starke Reize zwar abhängig von der Zeit, aber nicht in erkennbaren längeren oder kürzeren Zeitabschnitten, welche ebenfalls proportional der Reizstärke wären.

4. Die Nacherregung wird durch einen Reiz, welcher ein Nachbargebiet trifft, ausgelöscht.

Dieses letzte Gesetz ergebe möglicherweise neue therapeutische Verwendungsmöglichkeiten. - Auf Grund dieser Versuche gibt dann Richter noch einige therapeutische Hinweise für übererregte, $d . h$. also durch zu starke oder zu häufige Reize in Anspruch genommene Nerven, und für solche, die in einen Reizzustand getreten sind, welcher ihre normalen Erregungsgesetze umkehrt.

Ehe ich nun zu meinen eigenen Versuchen komme, möchte ich an dieser Stelle Herrn Prof. Foerster für die bereitwillige Förderung meiner Untersuchungen meinen Dank aussprechen, ebenso Herrn 
Dr. Goldberg für die liebenswürdige Unterstützung bei den Experimenten.

Ich habe nun festzustellen versucht, wie weit die anfangs beschriebene Wirkung des Leducschen Stromes auch für den Sinnesnerven zutrifft, und bin auf der Grundlage der Richterschen Gesetze namentlich die der Nacherregung und Erholung schienen mir bedeutungsvoll - unter Anwendung der von ihm angegebenen Elektroden (siehe oben) und unter genauer Einhaltung seiner Versuchsanordnung folgendermaßen vorgegangen: Zunächst kurze Wiederholung der Richterschen Versuche am N. opticus mit galvanischem Strome.

Anode am Auge, Kathode anal.

Stromstärke 0,2 M. A.: Weder bei Stromschluß noch bei Unterbrechung Lichtempfindung.

Stromstärke 0,5 M. A.: Bei Stromschluß Lichtblitz, bei Unterbrechung Dunkelempfindung; während der Dauer des Stromschlusses flackernde Lichterscheinung.

Stromstärke 0,8 M. A.: Dasselbe Resultat wie oben, etwas stärker.

Stromstärke 1,0 M. A.: Bei Schließung Lichtempfindung, während der Dauer der Durchfließung nichts, bei Unterbrechung Dunkelempfindung und sensible Empfindung am Anus.

Stromstärke 2,0 M. A.: Dasselbe Resultat, nur war die Lichtempfindung intensiver und die sensible Empfindung am Anus stärker.

Bei zehnmaliger rasch hintereinander erfolgender Öffnung und Schließung des Stromes, wobei die höchste erreichte Stromstärke 0,9 M. A. ist, werden 10 bläulich-violette Scheiben gesehen, sensible Erscheinungen nicht verspürt.

Eine Abweichung von den Richterschen Mitteilungen ist also insofern festzustellen, als bei Stromstärken von 1,0 M. A. und darüber während der Stromdauer eine Lichtempfindung nicht beobachtet wurde.

Bei ganz schwachem sinusoidal-faradischen Strome nicht so intensive Lichterscheinungen wie vorher, während der ganzen Durchfließungszeit, wie eine Kette von hellen und dunklen Perlen. Bei Unterbrechung keine Sensation am Auge oder am Anus. Bei etwas stärkerem sinus.-farad. Strome Verstärkung der Lichterscheinung, unangenehmes Brennen im Auge. Beim Stromschluß sensible Empfindung am Anus, bei Unterbrechung keinerlei Empfindung.

Kathode am Auge, Anode anal. Galvanisch.

Stromstärke 0,4 M.A.: Bei Schließung sensible Empfindung am Anus, bei Unterbrechung nichts.

Stromstärke 0,6 M. A.: Bei Schließung sensible Empfindung am Anus, bei Unterbrechung kleiner Lichtblitz. 
Stromstärke 1,0 M. A.: Bei Schließung sensible Empfindung am Anus, mit gleichzeitiger Lichterscheinung, während der Durchfließung nichts, bei Unterbrechung wieder ein Aufblitzen, jedoch schwächer wie bei der Stromschließung.

Leducscher Strom.

A node a m A uge, Ka thode a nal. Motor voll laufend. Periode: $1 / 2$.

Stromstärke 0,2 M. A.: Bei Schließung kleiner Lichtblitz, etwas Brennen im Auge, bei Unterbrechung Dunkelempfindung; während der Schließungsdauer keire Empfindung.

Stromstärke 0,7 M. A.: Bei Schließung kleiner Lichtblitz und Sensation am Anus, bei Unterbrechung Dunkelempfindung. Bei häufigem Schließen und Öffnen des Stromes stets nur Lichtempfindung bei Stromschluß.

Stromperiode : $3 / 4$.

Stromstärke 1,0 M.A.: Bei Stromschluß Lichtblitz, während des Durchfließens leichtes Brennen am Augenlid, bei Unterbrechung Dunkelempfindung und sensible Empfindung am Anus.

Strom periode : $1 / 4$.

Stromstärke 0,2 M. A.: Bei Schließung Spur eines Lichtblitzes, bei Unterbrechung nichts.

Stromstärke 0,5 M. A.: Bei Schließung Lichtblitz, begleitet von ziehendem Schmerz im Auge und im Kopfe, bei Unterbrechung Dunkelempfindung.

Strom periode $1 / 10^{\circ}$

Stromstärke 0,1 M. A.: Bei Schließung ziehender Schmerz im Auge und Andeutung von Helligkeit, bei Unterbrechung zunächst geringe Dunkelempfingung, bei Wiederholung keine Helligkeits- oder Dunkelempfindung mehr. Bei geringer Verstärkung des Stromes verstärkt sich die schmerzhafte Empfindung, bei Unterbrechung wie vorher Dunkelempfindung.

Stromperiode $1 / 100$.

Stromstärke zunächst kaum ablesbar.

Bei Verstärkung auf Stromstärke 0,1 M. A. tritt starker ziehender Schmerz im Auge und der zugehörigen Kopfseite auf, der während der Duchfließungsdauer anhält. Lichtempfindungen bei Schließung und Unterbrechung fehlen völlig.

Zusammenfassend ist also die Wirkung des L e $\mathrm{d} u \mathrm{c}$ schen einsteigen den Stromes bei Stromperioden mit langer Schließungsdauer eine dem galvanischen Strom ähnliche, während bei Stromperioden mit kurzer Schließungsdauer $(1 / 10,1 / 100)$ die Erregung des Sinnesnerven zurücktritt gegenüber der sehr starken Erregung der sensiblen Nerven. Dieses Verhalten ist darauf zurückzuführen, daß bei langer Schließungsdauer der Stromablauf dem des galvanischen Stromes in einer Reihen- 
folge von rhythmisch erfolgenden galvanischen Einzelerregungen entspricht, während bei kurzen Stromperioden der außerordentlich rasch eintretende Stromabfall dem Strom die gleiche, sensible Nerven erregende Komponente erteilt wie der faradische Strom. Gemäß dem Du-Bois-Raymondschen Gesetz muß auch die Schnelligkeit des Anwachsens und Abfallens des Stromgefälles bei der kurzen Stromschließungszeit von $1 / 100$ Periode einen besonders wirksamen und daher schon bei schwachen Stromstärken kaum erträglichen Reiz abgeben.

Die entsprechenden Versuche mit Leducschem aussteigenden Strome (Kathode am Auge, Anode anal) haben bei der gleichen Versuchsanordnung keine Abweichung von den analogen Richterschen Mitteilungen ergeben. Auch bei diesen Untersuchungen traten bei Stromperioden mit kurzer Schließungsdauer optische Erscheinungen vor den oft recht starken sensiblen Empfindungen völlig in den Hintergrund.

Es folgten dann Versuche über die Wirkung des L e d u c schen Stromes auf den N. opticus bei längerer Durchfließungsdauer. Versuchsanordnung war folgende: Anode am Auge, Kathode anal; Motor voll laufend; Stromperiode $3 / 4$. Zunächst Feststellung der Reizschwelle für Lichterscheinungen; dieselbe lag bei 0,2 M. A. Der kurz zuvor ausgeführte Versuch, die Reizschwelle für rein galvanischen Strom zu ermitteln, hatte das gleiche Resultat ergeben. Danach 5 Minuten Stromdurchfließung bei 1,0 M. A. Dabei zeigte sich anfangs ein leichtes Brennen auf dem Augenlid, das bald geringer wurde und nach etwa 50 Sekunden ganz verschwand. Während der ganzen Dauer der Stromdurchfließung keine Lichterscheinung und keine sensiblen Empfindungen, der Strom selbst wird nur ganz schwach als solcher und durchaus angenehm empfunden. Nach Ablauf von 5 Minuten Ausschleichen des Stromes, wobei lediglich geringe sensible Empfindungen am Anus ausgelöst werden. Erneute Feststellung der Reizschwelle ergibt jetzt eine Erhöhung derselben auf 0,4 bis 0,5 M. A. Nach weiterer Durchfließung mit 1,0 M. A., 5 Minuten lang, liegt die Reizschwelle bei 0,6 M. A.

Die gleichen Versuche mit aussteigendem Leducschen Strome bei längerer Durchfließungsdauer ergaben ebenfalls eine entsprechende Erhöhung der Reizschwelle.

Nach Beendigung der Versuche fand sich, je nach der Dauer der Stromdurchfließung, eine mehr oder minder starke Injektion der Conjunctiva, die spätestens in einer halben Stunde wieder zurückging. Das Sehvermögen war für die gleiche Zeitdauer gegen sonst insofern verändert, als man alle Gegenstände zunächst etwas undeutlich und wie durch einen leichten Schleier sah. Ob dieses Verhalten auf eine 
direkte Nachwirkung des Leducschen Stromes oder auf den Druck der mittels eines Gummibandes dem Bulbus fest angelegten Elektrode zurückzuführen ist, kann ich nicht mit Sicherheit entscheiden. Nach einer halben Stunde, meist sogar schon früher, war das Sehen wieder vollkommen normal; irgendwelche Nachwirkungen auf das normale Sehvermögen oder sonstige Schädigungen wurden nie beobachtet.

Zusammenfassend fand sich also bei längerer Durchfließung mit Leducschen einsteigenden sowie aussteigenden Strömen eine nicht unerhebliche Erhöhung der Reizschwelle für den N. opticus, oder mit anderen Worten eine Hypofunktion des Sinnesnerven, ein Vorgang, der durchaus analog ist der Hypästhesie des entsprechend behandelten peripheren Nervengebietes oder dem teilweisen Funktionsausfall des Zentralnervensystems bei der Ledu c schen Elektronarkose. Die oben angeführten Versuche erweitern somit auch die Gültigkeit der von Led uc und anderen Autoren festgestellten Wirkungen des unterbrochenen Gleichstromes niederer Spannung für den Sinnesnerven.

Zur Technik dieser Untersuchungen ist noch folgendes zu bemerken: Sollen Fehlerquellen in der Beobachtung und unliebsame Zwischenfälle vermieden werden, so müssen unbedingt die von Leduc angegebenen Richtlinien hinsichtlich der Versuchsanordnung eingehalten werden. Vor allem ist es erforderlich, daß die Elektroden fest und unverrückbar aufliegen, und daß der Strom langsam ein- und ausgeschlichen wird. Fehler dieser Art beeinträchtigen durch unvermittelt auftretende Lichterscheinungen die Beobachtung und verursachen zum mindesten höchst unangenehme sensible Empfindungen. Ein solcher Fehler - brüskes Ausschalten eines 10 Minuten bei 1,0 M. A. fließenden Stromes - verursachte einmal bei mir selbst einen mehrere Minuten währenden Zustand von elektrischer Narkose, fast genau derselben Art, wie ihn Led uc beschreibt ${ }^{18}$ ), nur daß eben noch Lichterscheinungen von strahlender Helligkeit (die Anode befand sich am Bulbus, die Kathode anal) hinzukamen. Es wird darüber noch an anderer Stelle berichtet werden.

Zum Schluß seien noch einige Hinweise für die therapeutische Nutzbarmachung des Leducschen Stromes am Auge gegeben. Wenn auch Richter sagt, am Auge wäre eine Domäne der Elektrotherapie, so ist doch in der Tat die Verwendung von elektrischen Strömen bei Augenerkrankungen bisher keine übermäßig große gewesen. v. Re uss ${ }^{19}$ ) verwendet zu diesem $Z$ wecke lieber den faradischen Strom, da er nicht mehr leiste als der galvanische und weniger komplizierte Apparate erfordere. Er rühmt die schmerzstillende Wirkung des Stromes bei Cyclitis und Iridocyclitis; schmerzhafte entzündliche Zustände in erblindeten Augen ließen sich in manchen Fällen durch den Induktionsstrom beseitigen, so da $\beta$ die Enucleation des Bulbus vermieden oder 
wenigstens auf einige Zeit hinausgeschoben werden konnte. Auch cyclitische Präcipitate auf der hinteren Hornhautfläche sowie Glaskörpertrübungen wurden mitunter schnell und günstig beeinflußt. Besonders gute Resultate ${ }^{20}$ ) beobachtete er bei Lichtscheu. Fehr ${ }^{21}$ ) bestätigt die Angaben von Reuss, ist jedoch sonst kein Enthusiast. Sile ${ }^{10}$ ) sah bei galvanischer Behandlung hysterischer Augenleiden gute Erfolge. Am wenigsten einer Behandlung zugänglich waren bisher die Erkrankungen des Sehnerven selbst, doch wird bei der einfachen (nicht bei der neuritischen) Sehnervenatrophie von verschiedenen Augenärzten bereits die Galvanisation empfohlen ${ }^{22}$ ). Wenn auch die Erfolge dieser Behandlungsmethode keine sehr bedeutenden sind, so ließ sich doch zuweilen durch sie eine objektiv nachweisbare Besserung der Sehkraft erzielen. Die Anwendung der Elektrotherapie erscheint hier um so berechtigter, als wir es hier mit einem Leiden zu tun haben, dem wir sonst völlig machtlos gegenüberstehen.

Für alle diese Erkrankungen des Auges erscheint somit unter Berücksichtigung vorstehender Untersuchungen eine Anwendung des Led u c schen Stromes indiziert. Nach den experimentell gewonnenen Resultaten sollte man erwarten, daß zum mindesten Lichtscheu, schmerzhafte Augenleiden und funktionelle Reizerscheinungen am Auge durch ihn günstig beeinflußt werden können. $\mathrm{Ob}$ er in der Tat hierbei und vielleicht auch bei den Erkrankungen des Sehnerven selbst mehr leistet als die bisher verwendeten Ströme, muß natürlich erst noch an pathologischen Fällen eingehend geprüft werden.

\section{Literaturverzeichnis.}

1) Leduc, Studie über intermittierende Gleichströme von niedriger Spannung. Arch. d'électr. méd. Nr. 129. 1903. - 2) Led u c, Die elektrische Narkose. Zeitschr. f. Elektrotherap. Nr. 11. Nov. 1903. ${ }^{3}$ ) D us cha k, E. Th., Über Leducsche Ströme. Zeitschr. f. phys. u. diät. Ther. 23, H. 10. 1919. - $\left.{ }^{4}\right)$ Erdmann, Die Anwendung der Elektrizität in der praktischen Medizin. Leipzig 1860. 5) Schlie phake, Zur Kenntnis der Einwirkung des galvanischen Stroms auf das menschliche Auge. Arch. f. d. ges. Physiol. 8, 565. - ${ }^{6}$ ) M üller, G. E., Uber die galvanische Gesichtsempfindung. Zeitschr. f. Psychiol. u. Physol. d. Sinnesorg. 14, 329ff. - 7) Wilbrandt und Saenger, Die Neurologie des Auges. Wiesbaden 1904. Bd. III, S. $293 \mathrm{ff}$. - $^{8}$ ) v. Helmholtz, A., Handbuch der physiologischen Optik. 3. Aufl. - $\left.{ }^{9}\right)$ Tarducci, A., Verschiedene Wirkung des aufsteigenden und des absteigenden Stromes auf das Gesichtsfeld. Ref. aus Annali di Oftalmologia 1897, H. 6. - ${ }^{10}$ ) Silex, Klinisches und Experimentelles aus dem Gebiete der Elektrotherapie bei Augenkrankheiten. Arch. f. Augenheilk. 37, H. 2, S. $127 \mathrm{ff} .-{ }^{11}$ ) Brenner, Untersuchungen und Beobachtungen auf dem Gebiete der Elektrotherapie. Leipzig 1868/69. $\neg{ }^{12}$ ) Erb, Wilh., Handbuch der Elektrotherapie. Leipzig 1882, S. 105. - 13) Richter, Ed., Neue Gesetze der Erregung von Sinnesnerven und zwei neue Elektrodenpaare für das $\mathrm{Ohr}$ und das Auge. Monatsschr. f. Ohrenheilk. 1900, H. 12, S. 454. - ${ }^{14}$ ) Richter, Ed., Die biologischen Gesetze der Nervenerregung in zentrifugalen und zentripetalen Nerven. 
272 H. Taterka: Untersuchungen am Nervus opticus mit Leducschem Strome.

Zeitschr. f. d. ges. Neurol. u. Psychiatr. 48. - 15) Hoff man n und Banzel, Untersuchungen über den elektrischen Geschmack. Arch. f. d. ges. Physiol. 66, $215 \mathrm{ff}$. - ${ }^{16}$ ) von Zeynek, Über den elektrischen Geschmack. Zentralbl. f. Physiol. 12, H. 19, S. 617. - ${ }^{17}$ ) He u man n, G., Einige Untersuchungen, betreffend den elektrischen Geschmack. Zeitschr. f. med. Elektrol. 13. 1911. - ${ }^{18}$ ) Leduc, Die elektrische Hemmung der Hirntätigkeit beim Menschen. Zeitschr. f. Elektrotherap. u. physikal. Heilmethoden H. 2, Februar 1903. $-{ }^{19}$ ) von Reuss, A., Neue Erfahrungen über die Elektrotherapie entzündlicher Augenkrankheiten. Arch. f. Ophthalmol. 46, 398ff. 1898. - ${ }^{20}$ ) von Reuss, A., Meine Methode der elektrischen Behandlung entzündlicher Augenleiden. Wien. med. Wochenschr. 1913, Nr. 39. - ${ }^{21}$ ) Fehr, Die Anwendung der Elektrizität in der Augenheilkunde. Leipzig 1911. - ${ }^{22}$ ) Kowarschik, Elektrotherapie. Berlin 1920, S. 281. 